\section{Pilonidal Disease can be Treated by Dermatologists with Crystallised Phenol in Outpatient Clinics}

\section{Sir,}

Pilonidal disease, hidradenitis suppurativa, acne conglobata, and dissecting cellulitis of the scalp are the members of follicularocclusion tetrad with similar pathophysiology, which is initiated by follicular occlusion in apocrine gland bearing areas. ${ }^{1}$ Interestingly, while the treatment of hidradenitis suppurativa, acne conglobata, and dissecting cellulitis has been undertaken by dermatologists, the treatment of pilonidal disease has been left to general surgeons. However, pilonidal disease can also be treated by dermatologists with crystallised phenol using a minimally invasive technique in outpatient clinics. ${ }^{2}$ Hereby, we would like to explain crystallised phenol technique in detail in order to encourage dermatologists to treat pilonidal disease. The detailed visual description of crystallised phenol technique in the treatment of pilonidal disease is depicted in Figure 1.

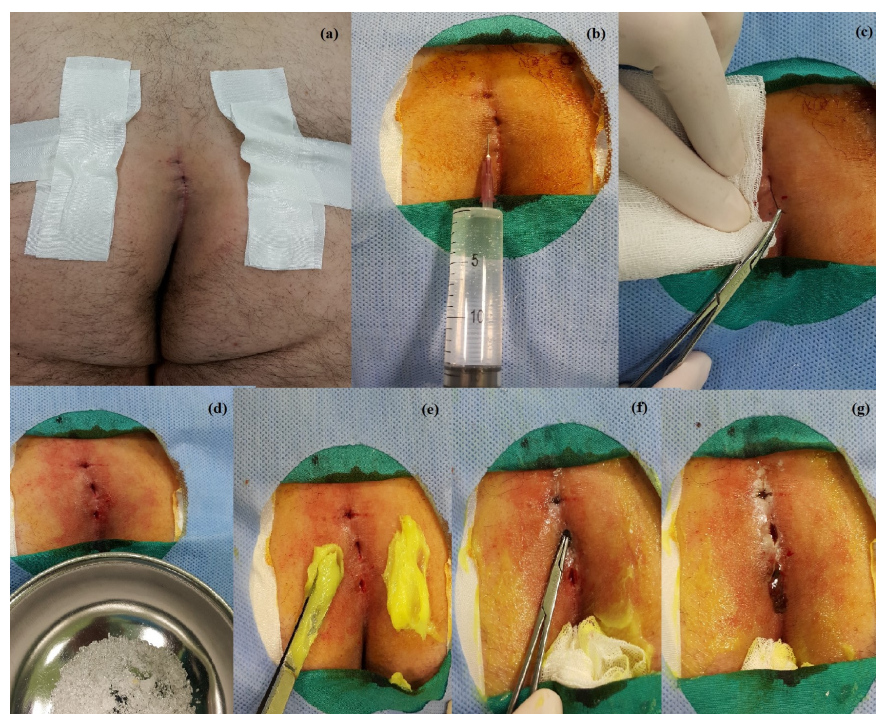

Figure 1: (a) Intergluteal area is exposed with the help of adhesive bandages which pull buttocks apart. Three pilonidal pits in the intergluteal sulcus are visible.

(b) The intergluteal region is prepped and draped in a sterile fashion. Povidone-iodine $(7.5 \%)$ is preferred as antiseptic scrub. Five mililiters of $2 \%$ lidocaine solution is applied subcutaneously to maintain local anesthesia in order not to cause pain during hair removal from pilonidal pits.

(c) Hair is gently pulled out from the pilonidal pits with a mosquito clamp. Hair should not be harshly plucked or pulled out. Applying excessive force may cause bleeding. If bleeding occurs, please place a gauze pad and compress itgentyluntil the bleeding stops.

(d) Crystallised phenol (Phenol, Emprove $₫$, Merck, Darmstadt, Germany) resembles white sugar granules. Crystallised phenol is in solid form in room temperature. As soon as it gets into contact with the skin, crystallised phenol melts into liquid form, and thus causes a chemical burn with small white blisters. Therefore, a facemask, eye-protection glasses, surg- ical gloves and a gown with long sleeves should be worn prior to phenol application. Dermatologists should never neglect wearing the self-protection equipment.

(e) In order to protect the skin from the chemical burn effect of crystallised phenol, $0.2 \%$ nitrofurazone ointment (Furacin, Zentiva) is spread with the back of a pincette onto the gluteal region. However, other topical creams such as $2 \%$ sodium fusidate (Fucidin) may also be applied.

(f) Liquid phenol may leak to the perianal area; therefore, the anus should be covered and protected with a gauze pad. This gauze pad can be soaked into a soluble dressing such as $0.2 \%$ nitrofurazone ointment. Afterwards, pilonidal pits are meticulously filled with crystallised phenol. Crystallised phenol causes chemical debridement within the pilonidal sinus pits. (g) As the crystallised phenol causes chemical debridement within the pilonidal pits, dark brown leakage occurs from the sinus orifices. There is no universally accepted amount of crystallised phenol to fill pilonidal pits or number of application cycles. Therefore, the dermatologists should apply crystallised phenol until the dark brownish discharge decreases. For the time being, experience is the key to decide for the minimum amount of crystallised phenol needed for the best treatment outcome. A gauze pad should be placed on the intergluteal region after the phenol application. The gauze pad should absorb the debris caused by the chemical debridement. The patients should daily wash the treated area with hot water. The closure of pilonidal pits without any discharge is accepted as healing. Complete healing generally occurs inbetween seven to thirty days.

The treatment of pilonidal disease with crystallised phenol has many advantages. This technique is minimally invasive; moreover, learning curve is extremely short. Only one-time application is enough to learn the whole procedure. There is no need for operating room settings. Dermatologists can apply crystallised phenol to their patients with pilonidal sinus in their outpatient clinics. Furthermore, patients can get to work after the procedure immediately. Possible postoperative complications, such mild pain, purulent discharge, and abscess formation, can be treated with non-steroidal anti-inflammatory drugs and antibiotics. ${ }^{2}$ Moreover, referring the patient to a general surgeon is always an available option.

The post-treatment cosmetic results are excellent, as there are no incisions or stitches after the procedure. Phenol treatment has a $30-40 \%$ failure rate with multiple and suppurative pilonidal disease. However, patients who have limited disease (1-3 pilonidal pits) have higher success rates. ${ }^{3}$ Our two-year follow-up of 50 patients with pilonidal disease treated with one-time application of crystallised phenol revealed $80 \%$ complete healing rate. Therefore, we encourage our dermatology specialist colleagues to treat patients diagnosed with pilonidal disease with crystallised phenol in their outpatient clinics.

\section{CONFLICT OF INTEREST:}

Authors declared no conflict of interest.

\section{AUTHOR'S CONTRIBUTION:}

MEY: Conception, design, drafting the manuscript and approval forpublication.

\section{REFERENCES}

1. Vasanth V, Chandrashekar BS. Follicular occlusion tetrad. Indian Dermatol Online J 2014; 5:491-3.

2. Yuksel ME. Pilonidal sinus disease can be treated with crystallized phenol using a simple three-step technique. 
Acta Dermatovenerol Alp Pannonica Adriat 2017; 26:15-7.

3. Kober MM, Alapati U, Khachemoune A. Treatment options for pilonidal sinus. Cutis 2018; 102:23-9.

Mehmet Eren Yuksel

Intensive Care Unit, Yildirim Beyazit University School of Medicine, Ankara, Turkey
Correspondence to: Mehmet Eren Yuksel, Yildirim Beyazit Universitesi Tip Fakultesi Hastanesi Üniversiteler Mahallesi Ihsan Doğramacı Bulvari 06800 Ankara, Turkey

E-mail: doctormehmeteren@yahoo.com

Received: August 16, 2019; Revised: August 17, 2019;

Accepted: August 09, 2020

DOI: https://doi.org/10.29271/jcpsp.2020.07.772

$\bullet \bullet \bullet \bullet \bullet \bullet \bullet \bullet \bullet \bullet$ 